\title{
Reprints and Microfilms of Certain Periodicals
}

$\mathrm{T}$ He A.L.A. Committee on Aid to Libraries in War Areas has found it necessary to microfilm or reprint certain out-of-print wartime issues of American periodicals in connection with its program of distribution of periodicals to foreign libraries.

In cases where the committee needed twenty or more copies of unavailable issues, they decided to reprint by photographic reproduction in reduced size, i.e., four pages of the original on one page of the reproduction. Page size of the reproduction remains the same as that of the original in order to facilitate binding. Of these reproductions, editions of one hundred copies each were ordered, so that, after the committee's needs are satisfied,

there will be from forty to eighty copies of each reproduced periodical available for sale. The first list below shows reprinted periodicals which may be obtained from Edwards Brothers, Ann Arbor, Mich.

The second list indicates periodical issues being microfilmed for the committee, most of which will be available in microfilm copy from University Microfilms, Inc., Ann Arbor, Mich. University Microfilms is also interested in arranging for reproduction of other out-of-print issues of these journals if there is a demand for them.

It seems likely that there will be libraries both at home and abroad which will be interested in purchasing some of these reprints or microfilms to complete their periodical files.

\section{List of Reprints by Edwards Brothers in Ann Arbor, Mich.}

American Heart Journal, v. 24, no. I, July 1942; v. 24 , no. 2 , Aug. 1942 ; v. 26 , no. 1, July 1943; v. 26 , no. 3 , Sept. 1943.

American Journal of Obstetrics \& Gynecology, v. $4 \mathrm{I}$, no. 4 , Apr. $194 \mathrm{I}$; v. $4 \mathrm{I}$, no. 6 , June $194 \mathrm{I}$; v. 42 , no. 2 , Aug. 1941 ; v. 43 , no. 1, Jan. 1942; v. 43 , no. 4 , Apr. 1942; v. 45 , no. 3 , Mar. 1943; v. 45 , no. 5 , May 1943 ; v. 46 , no. 3 , June 1943 . American Journal of Surgery, v. 51, no. 2, Feb. 1941.

American Journal of Tropical Medicine, v. 23, no. I, Jan. 1943 \& supplement.

American Political Science Reviequ, ${ }^{1}$ v. 35, no. r, Feb. r94r.

Archives of Internal Medicine, v. 67, Jan.June r94r.

1 Extra copies are being handled by the publisher, American Political Science Association, Northwestern University, Evanston, III.
Archives of Neurology \& Psychiatry, v. 44, July-Dec. 1940; v. 45, Jan.-June 1941.

Archives of Ophthalmology, v. 25, Jan.-June, r94r.

Archives of Otolaryngology, v. 33, Jan.-June I94.

Endocrinology, v. 26, nos. 4-5, April-May, I940.

Journal of Bacteriology, v. 4I, Jan.-June 194r. Journal of Infectious Diseases, v. 67, no. 3, Nov.-Dec. 1940.

Journal of Laboratory \& Clinical Medicine, v. 27 , nos. $6,7,9$, 10, 11, Mar., April, June, July, Aug. 1942 ; v. 28 , nos. $1,4,7,8$, Oct. 1942, Jan., April-May 1943.

Journal of Urology, v. 45, Jan.-June r94r.

War Medicine, v. $1,194 \mathrm{r}$; v. 2 , nos. $1-4$, Jan.Feb.-July-Aug. 1942. 


\section{List of Microfilms by University Microfilms, Inc., Ann Arbor, Mich.}

Acta Americana, v. 1, no. r, Jan. 1943 .

American Heart Journal, v. 21, no. r, Jan. r94I ; v. 24, no. 4, 5, 6, Oct., Nov., Dec. 1942; v. 25 , no. 3, Mar. 1943; v. 29, no. 2, Feb. 1945 . American Journal of Digestive Diseases, v. 9, no. 5, May 1942; v. 1o, no. 4, April r943.

American Journal of Diseases of Children, v. 59, Jan.-June 1940.

American Journal of Obstetrics \& Gynecology, v. 40 , no. I, July r 940 ; v. 4 r, no. I, 2, 3, Jan., Feb., Mar. 1941 ; v. 42, no. I, 3, 4, July, Sept., Oct. I $94 \mathrm{I}$; v. 43 , no. 5 , May 1942 ; v. 46 , no. 6 , Dec. 1943 ; v. 47 , no. 5 , 6, May, June 1944 ; v. 51, no. 2, Feb. 1946.

American Journal of Ophthalmology, v. 26, no. I, 5, Jan., May 1943.

American Journal of Orthodontics, v. 28, no.

4, April r942.

American Journal of Roentgenology and Radium Therapy, ${ }^{2}$ v. 46 , no. I, July $194 \mathrm{r}$; v. 50 , no. 4 , Oct. 1943; v. 52, no. 5, 6, Nov., Dec. 1944.

American Journal of Surgery, v. 47, no. 2, 3,

Feb., Mar. 1940.

American Meteorological Society Bulletin, v. 24 , no. I, Jan.' 1943.

American Naturalist, ${ }^{2}$ v. 75 , no. 1-3, Jan.-Feb.

-May-June r94r; v. 8o, no. 5, Mar. 1946.

American Society of Agronomy Journal, v. 35, no. 1, Jan. 1943.

Anesthesiology, v. 4, no. 2, 3, 4, 5, 6, Mar.-Nov. I 943 ; v. 5 , no. I, Jan. 1944.

Archives of Dermatology, v. 42, July-Dec. 1940.

Archives of Neurology and Psychology, v. 43, Jan.-June 1940.

Archives of Ophthalmology, v. 23, Jan.-June I940.

Archives of Pathology, v. 29, Jan.-June r940. Archives of Surgery, v. 40, Jan.-June 1940.

Books Abroad, v. 15, no. 1, Jan. 1941; v. 19, no. 3, July 1945 .

Botanical Revieq, v. 6, no. 2, Feb. r940.

Chemical Reviequs, v. 27, Aug.-Dec., 1940.

Far Eastern Quarterly, v. I, no. 3, May 1942.

Food Research, v. 8, no. I, Jan. 1943.

General Electric Revieq, v. 46, no. 12, Dec. 1943.

Journal of Abnormal and Social Psychology, v. 36 , no. $\mathrm{I}$, Jan. $194 \mathrm{I}$.

Journal of Accountancy, v. 73 , no. 5, May 1942; v. 75, no. I, 3, 4, 5, 6, Jan., Mar.-June 1943; v. 76 , no. 1 , July 1943 .

${ }^{2}$ Permission to sell copies pending.
Journal of Aviation Medicine, v. 12, no. I, 2, Mar., June r94r.

Journal of Chemical Education, ${ }^{3}$ v. 18, no. 2, Feb. 1941.

Journal of Documentary Reproduction, ${ }^{3}$ v. 5 , no. 3, 4, Sept., Dec. 1942.

Journal of Economic Entomology, ${ }^{2}$ v. 34, no. 2, 4, April, Aug. 194r.

Journal of Immunology, v. 40, Jan.-April r94r. Journal of Laboratory and Clinical Medicine, v. 27 , no. 2 , Nov. 1941; v. 27, no. 8 , May 1942. Journal of Oral Surgery, ${ }^{2}$ v. 1, no. 1, 2, Jan.. April 1943.

Journal of Pediatrics, ${ }^{2}$ v. 21, no. 2, Aug. 1942; v. 28 , no. 5 , May 1946 .

Journal of Pharmacology and Experimental

Therapeutics, v. 7r, Jan.-April r94r.

Journal of Thoracic Surgery, ${ }^{2}$ v. 14, no. I,

Feb. 1945; v. 15, no. 1, Feb. 1946.

Marine Engineering and Shipping Revieq, v. 47, no. 1, 3, 4, 8, 9, Jan., Mar., April, Aug., Sept. r942; v. 48 , no. $1,2,3,6,7,8$, 9, ro, Jan.Mar., June-Oct. r943; v. 49, no. 2, 3, Feb., Mar. 1944.

Petroleum Engineer, v. 14, no. 1, 2, 3, 4, Oct. 1942-Jan. 1943; v. 16, no. 4, Jan. 1945.

Physiological Reviequs, v. 25, no. 1, 2, Jan., April 1945.

Power Plant Engineering, v. 46 , no. 3, 7, 10, Mar., July, Oct. 1942; v. 47 , no. 2, 6, Feb., June 1943; v. 48 , no. I, Jan. 1944.

Public Health Nursing, v. 33, no. I, Jan. 1941 ; v. 35 , no. 4 , April 1943 .

Quarterly Journal of Studies on Alcohol, ${ }^{2} \mathbf{v}$. I, no. 2, Sept. 1940.

Railway Age, v. 112, no. 1, 15, Jan. 3, April II, I942; v. II3, no. 21 , Nov. 21 , I942; v. I14, no. II, 12, 24, Mar. 13, 20, June 12, r943; v. Ir 5 , no. $5,19,21,25$, July 31 , Nov. 6,20 , Dec. 18, 1943; v. 116, no. $2,3,5,6,7$, 10, 11, 12, Jan. 8, 15, 29, Feb. 5, 12, Mar. 4, 11, 18, 1944 .

Review of Economic Statistics, v. 23, no. 2, May 194r; v. 25, no. 2, May r943; v. 27, no. 4, Nov. 1945; v. 28 , no. I, Feb. 1946.

Sequage Works Journal, v. 13, no. I, Jan.-Feb. r94r.

Social Research, ${ }^{2}$ v. 7, no. 2, 4, May, Nov. r940; v. 9, no. 3, Aug. 1942; v. Ir, no. 2, May 1944; v. 13, no. 1, Mar. 1946.

Surgery, ${ }^{2}$ v. 14, no. 4, Oct. 1943.

Yale Law Journal, ${ }^{2}$ v. 55, no. 1, Dec. 1945.

3 Other out-of-print issues also available. 